\title{
Thermal Effect Analysis of Hot flow Manifold Made for Industrial Automated Washing Machine
}

\author{
Pooja G Ekade ${ }^{1}$, Dr. Rachayya.R.Arakerimath ${ }^{2}$ \\ Mechanical Department, G. H Raisoni COEM/ Pune University, India
}

\begin{abstract}
Automated Manifold acting in component processing machine is developed with thermal effect assessment. Manifold to perform transfer of fluid and air through optimized inlet piping with pneumatically sliding whole assembly. Vertical and horizontal mounting feasibility for this assembly gives perfect solution to make transfer of fluid in any position. Non reachable geometrical surfaces and complex shapes are to be covered for washing and drying which are already with bur, oil and dirt. This manifold is to be installed in SPM washing machine. Heated air and heated fluid intake gives perfect required supply through output temperature in medium to perform cycle operations in SPM. Considered output temp of medium is at least $45^{\circ} \mathrm{C}$. Manifold sliding design parameters are developed with considering all engineering terms. Manifold with optimized thermal effective solution to achieve minimum $45^{\circ}$ C. Flow Analysis is performed with Rate of heat transfer through this dedicated manifold and heat fluxes on manifold body is validated in this work.
\end{abstract}

Keywords: Cylinder Block, Manifold, SPM (Special purpose Machine), Thermal analysis.

\section{Introduction}

After performing machine operations on any component or for just cleaning purpose, the components have to be washed. Just after washing and before proceeding component to assembly level, it should be dried and free from water on the surface. But if they have some complex cavities (like oil galleries in Cylinder Block) it is not easy to dry or wash these kinds of complex profile areas. For this, a system of nozzle is used which is responsible for washing and drying such complex profile cavities within components. And these portion are processed with the help of automated manifold, Manifold are designed generally in pipe structures and bends of pipes welded, in this research the manifold is considered with sliding assembly. In assembly pipe is also sliding to reach the nozzles till object.

\section{Automatic Washing Machine Specification}

An automatic component washing machine is shown in the below images. This machine is a special purpose machine which is used to wash and dry various components.

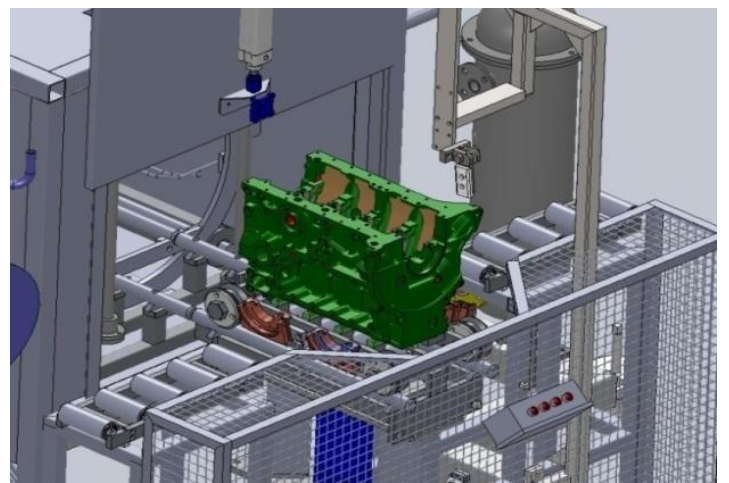

Figure 1: Component entering inside machine cabinet

1.1 Machine Specifications:-

Below table gives the specifications of automatic washing machine.

Utilities Required

\begin{tabular}{|l|l|l|}
\hline \multicolumn{2}{|c|}{ Consumption in Machine } \\
\hline 1 & Clean Tank & 300 litters water with (3-5\% washing chemical) \\
\hline 2 & Dirty Tank & 550 litters water with (3-5\% washing chemical) \\
\hline 3 & Compressed Air & 90 CFM @ 4.0BAR \\
\hline 4 & Power & $40 \mathrm{KW}, 3 \mathrm{PH}$ \\
\hline 5 & Compact band filter paper & $600 \mathrm{~mm}$ width paper, 20 Micron \\
\hline 6 & Filter Bag & 7 " $\times 20$ " filter bag, 10 Micron \\
\hline
\end{tabular}


Processes Parameters

\begin{tabular}{|l|l|l|l|}
\hline Sr No & Process Parameter & Required Value & Checking Method \\
\hline 1 & Spray Line Pressure & $12-16 \mathrm{Bar}$ & On Respective pressure guages \\
\hline 2 & Transfer Line Pressure & $0.5-2 \mathrm{Bar}$ & On Respective pressure guages \\
\hline 3 & Air Pressure & $4-4.5 \mathrm{Bar}$ & On Respective pressure guages \\
\hline 4 & Clean tank Temp & $55-65^{\circ} \mathrm{C}$ & On Respective TIC \\
\hline 5 & Dirty tank Temp & $55-65^{\circ} \mathrm{C}$ & On Respective TIC \\
\hline 6 & Clean Tank water level & In green zone & On Respective TIC \\
\hline 7 & Dirty Tank water level & In green Zone & On Respective TIC \\
\hline
\end{tabular}

\section{Objective Of The Reserch}

1.2 To Find out Solution to Maintain Temperature at the Outlet of the Manifold, so as to wash and dry the Component.

1.3 To Find out Effective Thermal Solution To Achieve Minimum $45^{\circ} \mathrm{C}$

1.4 To Optimize the Design of Manifold to Achieve Millipore value in Washing Machine.

\section{Implementation}

In this chapter most of the technical aspects of the parts and materials used in this thesis, and the basic settings used on the simulations will be described. Firstly, a review on the Finite Element Method (FEM) will be performed so that the process followed in every simulation can be explained.

\subsubsection{Working on Flow With FEM}

1.4.2 Creation of 3D model: Preparation of the model before taking it into FEM software such as elimination of bad geometry, simplification of unnecessary parts and improvement of contact regions

1.4.3 Basic Inputs in ANSYS: This information allow the model to work properly during simulation and will define the behavior of the parts during the analysis

1.4.4 FEM Inputs: These parameters control the computational time and the accuracy of results in the simulation. A special analysis of factors is performed in order to define them.

1.4.5 Simulation is carried out by software's.

1.5 Transient Thermal Analysis

A transient thermal analysis follows basically the same procedures as a steady-state thermal analysis. The main difference is that most applied loads in a transient analysis are functions of time.

1.6 Task in thermal Analysis

The procedure for performing thermal analysis involves building of model, applying loads and then reviewing results.

1.7 To Design Conceptual Model

\section{Methodology}

1.8 Proper Arrangements of Inlet and Outlet

1.9 Minimum Surface Area to Avoid Heat Losses

1.10Effect of Medium Temperature on Manifold Body

1.11 Application Feasibility Checking

1.12Effective heating medium supply calculations

1.13Validation on Ansys, thermal fluxes simulation

\section{Designed Manifold Structure And Parts}

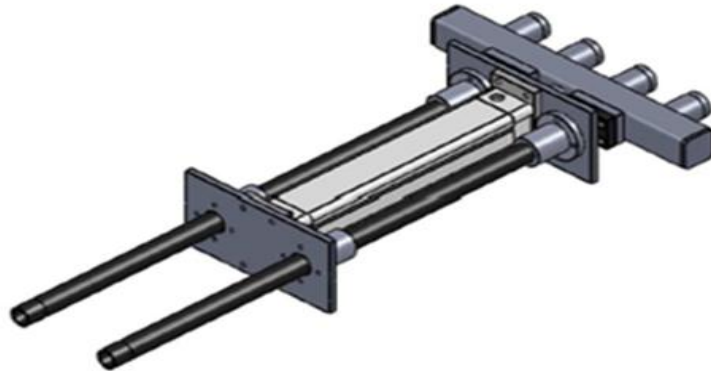

Fig 2: Pipe manifold with sliding assembly

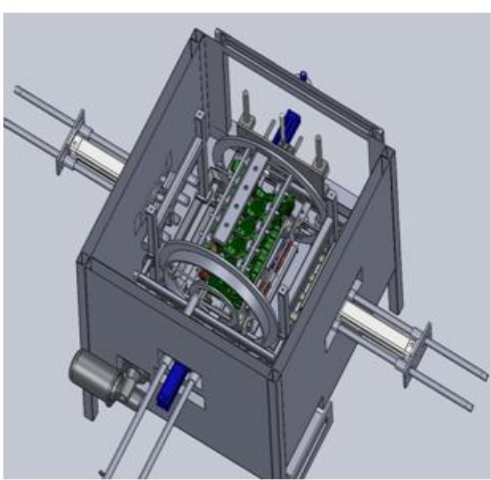

Fig 3: Installed manifold sliding assembly 


\section{Working With ANSYS To Analyze Thermal Phenomenon}

A thermal analysis calculates the temperature distribution and related thermal quantities in a systemor component. Typical thermal quantities of interest are:

1.14The temperature distributions

1.15The amount of heat lost or gained

1.16Thermal Gradients

1.17Thermal Fluxes

\subsubsection{How ANSYS Treats Thermal Modelling:-}

Only the ANSYS Multi-physics, ANSYS Mechanical, ANSYS Professional, and ANSYS FLOTRAN program support thermal analyses. The basis for thermal analysis in ANSYS is a heat balance equation obtained from the principle of conservation of energy. (For details, consult the Mechanical APDL Theory Reference.) The finite element solution we perform via Mechanical APDL calculates nodal temperatures, then uses the nodal temperatures to obtain other thermal quantities. The ANSYS program handles all three primary modes of heat transfer:

- Conduction,

- Convection,

- Radiation

7.1.2 Tasks in Thermal Analysis:-

- $\quad$ Build the model.

- Apply loads and obtain the solution.

- Review the results.

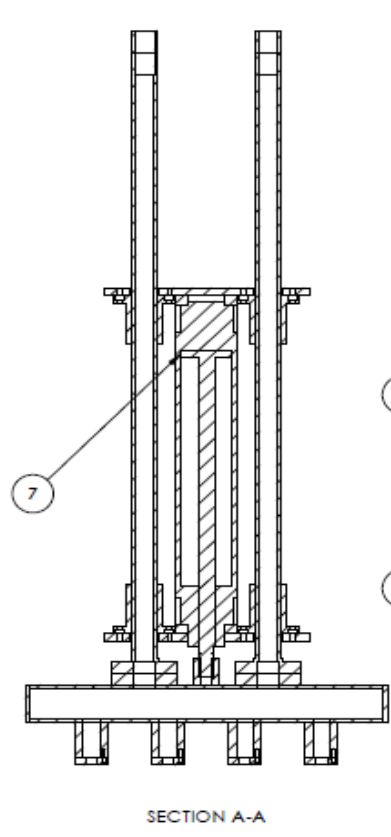

VIII. Details Of Manifold Assembly

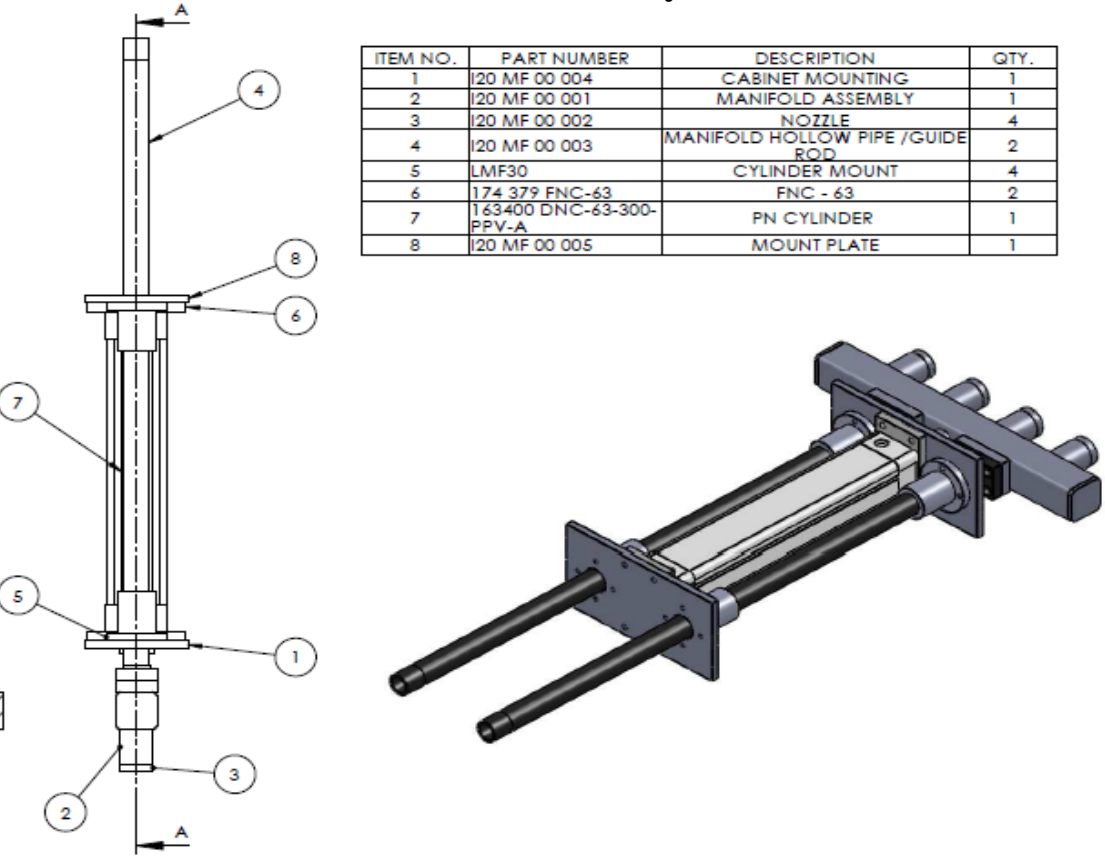

Fig 4: Details of proposed assembly

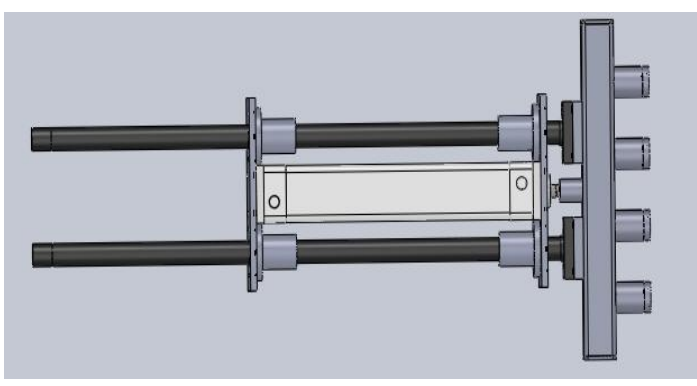

Fig 5: Closed inactive position

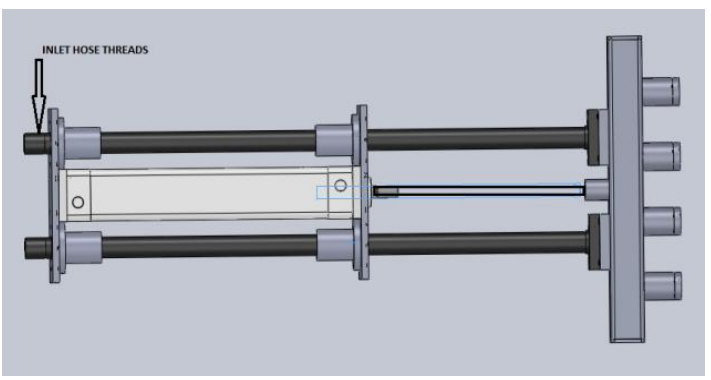

Fig 6: Open active position 


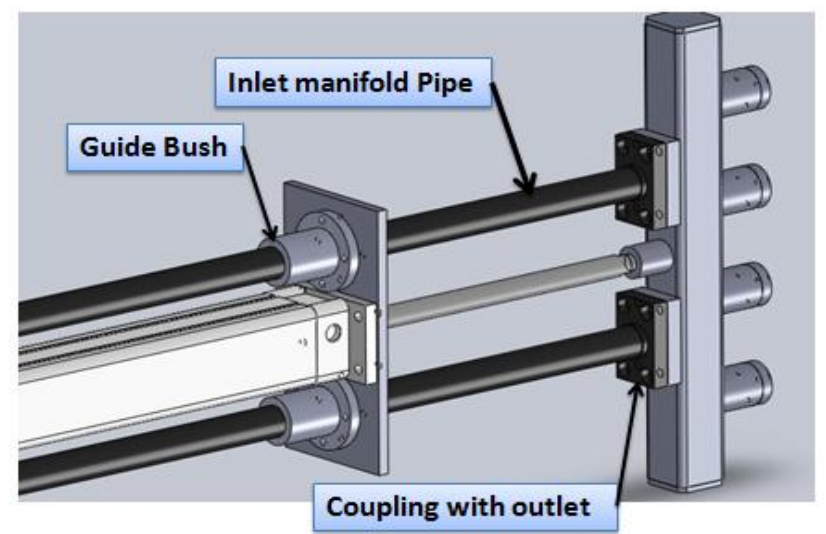

Fig 7: Inlet Pipe connection

\section{Analytical Assessment Of Manifold Component}

We have,

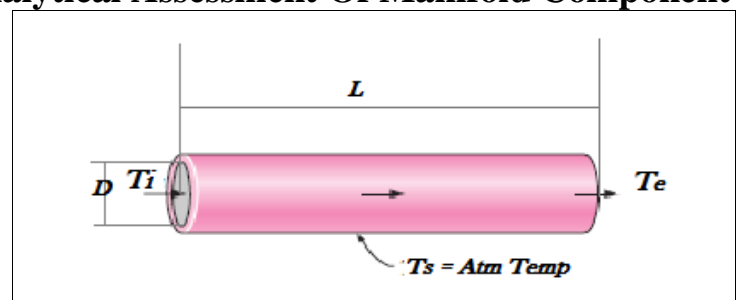

Fig 8: Analytical Design of Manifold

$\mathrm{Ti}=$ Inlet Temperature $=75^{\circ} \mathrm{C}$

$\mathrm{L}=$ Length of manifold $=1200 \mathrm{~mm}$

$\mathrm{D}=$ Inner Dia of manifold $=27.5 \mathrm{~mm}$

Material of Exhaust manifold is Stainless steel 304

The temperature at the exit of the manifold is given by)

$\mathrm{T}_{\mathrm{e}}=\mathrm{T}_{\mathrm{s}}-\left[\left(\mathrm{T}_{\mathrm{s}}-\mathrm{T}_{\mathrm{i}}\right) \exp \left(-\mathrm{hA}_{\mathrm{s}} / \mathrm{mC}_{\mathrm{p}}\right)\right]$

Where,

$\mathrm{T}_{\mathrm{s}}=$ Surrounding Temperature $($ atmospheric Temp $)=$ Constant $=30^{\circ} \mathrm{C}$ (Assumed)

$\mathrm{h}=$ Average heat transfer Coefficient $\left(\mathrm{W} / \mathrm{m}^{2}\right)$

$\mathrm{m}=$ Mass flow rate $(\mathrm{kg} / \mathrm{s})$

$\mathrm{C}_{\mathrm{p}}=$ Specific heat of water $(\mathrm{kJ} / \mathrm{kg})$

$\mathrm{A}_{\mathrm{s}}=$ Surface Area $=\pi \mathrm{DL}\left(\mathrm{m}^{2}\right)$

The fluid entering in to the manifold at $200 \mathrm{lit} / \mathrm{min}$

$\mathrm{m}=3.4 \mathrm{~kg} / \mathrm{s}$ (as $1 \mathrm{lit} / \mathrm{min}=0.017 \mathrm{~kg} / \mathrm{s})$

$\mathrm{A}_{\mathrm{s}}=\pi \mathrm{DL}$

$$
=0.104 \mathrm{~m}^{2}
$$

Now,

We have, $\mathrm{Q}=\mathrm{mC} \Delta \mathrm{T}$

Where $\Delta \mathrm{T}=\mathrm{T}_{\mathrm{i}}-\mathrm{Ts}=75-30=45^{\circ} \mathrm{C}$

$\mathrm{C}=40187 \mathrm{~kJ} / \mathrm{kg}$

Hence,

$\mathrm{Q}=640.61 \mathrm{~kJ} / \mathrm{s}$

Also we have,

$\mathrm{Q}=\mathrm{hA} \mathrm{s}_{\mathrm{s}}\left(\mathrm{T}_{\mathrm{i}}-\mathrm{T}_{\mathrm{s}}\right)$

Therefore,

$h=136.88 \mathrm{~W} / \mathrm{m}^{2}$

Putting all these values in equation (i) for $\mathrm{T}_{\mathrm{e}}$, We get,

$\mathrm{T}_{\mathrm{e}}=46.66^{\circ} \mathrm{C} \sim 47^{\circ} \mathrm{C}$

From the above Calculation We get the temperature at exit of the manifold is $47^{\circ} \mathrm{C}$.

And the minimum required fluid temperature at the outlet side of the manifold is around $40^{\circ} \mathrm{C}-45^{\circ} \mathrm{C}$.

Now, the rate of heat transfer through the manifold,

$\mathrm{Q}=\mathrm{mC}_{\mathrm{p}} \Delta \mathrm{T}$

$\mathrm{Q}=3.4 \times 4.187 \times(75-47)$

$\mathrm{Q}=398.6 \mathrm{~kJ} / \mathrm{s}$ 


\section{Thermal Stresses}

The thermal stresses acting on the manifold can be calculated by

$\sigma=\epsilon \mathrm{E}$

$=\Delta \mathrm{L} \mathrm{E} / \mathrm{L}$

Where,

$\Delta \mathrm{L}=$ Amount of thermal expansion in length

$=\alpha \Delta \mathrm{T} \mathrm{L}$

$\alpha=$ thermal expansion coefficient

$=16 \times 10^{-6} / \mathrm{K}$

$\Delta \mathrm{L}=0.0005 \mathrm{~m}$

$\mathrm{E}=$ modulus of elasticity $=190 \mathrm{GPa}$

Hence thermal stresses

$$
\sigma=79.17 \mathrm{MPa}
$$

Maximum uni-axial thermal stresses is given by,

$\mathrm{E}=190000 \mathrm{MPa}$

$\alpha=16 \times 10^{-6} / \mathrm{K}$

Maximum uni-axial thermal stresses is given by,

$$
\begin{aligned}
& \sigma_{x}=\frac{E \alpha}{(1-\vartheta)}\left(T_{i}-T_{e}\right. \\
& \mathrm{E}=190 \mathrm{GPa} \\
& \vartheta=\text { Poissions ratio }=0.3 \\
& \alpha=16 \times 10-6 / \mathrm{K} \\
& \text { Hence, }
\end{aligned}
$$

$$
\sigma_{x}=115.80 \mathrm{MPa}
$$

1.18Heat Losses in conduction and convections

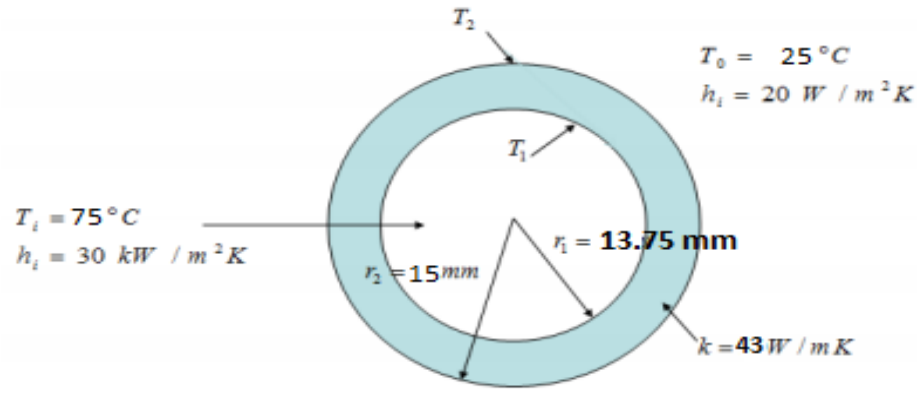

Fig 9: Cross section of manifold for analysis

$$
Q=2 \pi r_{1} L h_{i}\left(T_{i}-T_{1}\right)
$$

Hence,

$$
\left(T_{i}-T_{1}\right)=\frac{Q}{2 \pi r_{1} L}
$$

Similarly,5

$$
Q=2 \pi r_{2} L h_{o}\left(T_{2}-T_{o}\right)
$$

Hence,

$$
\left(T_{2}-T_{o}\right)=\frac{Q}{2 \pi r_{2} L h_{o}}
$$

$$
Q=\frac{2 \pi L k\left(T_{1}-T_{2}\right)}{\ln \left(r_{2} / r_{1}\right)}
$$

Hence,

$$
\left(T_{2}-T_{1}\right)=\frac{Q}{2 \pi L K / \ln \left(r_{1} / r_{2}\right)}
$$

Adding three equations on right column eliminates the wall temperatures gives, 


$$
Q=\frac{2 \pi L\left(T_{i}-T_{o}\right)}{\frac{1}{h_{i} r_{1}}+\frac{\ln \left(r_{2} / r_{1}\right)}{k}+\frac{1}{h_{0} r_{2}}}
$$

Hence heat loss per unit length

$$
\begin{aligned}
& \frac{Q}{L}=\frac{2 \pi\left(T_{i}-T_{o}\right)}{\frac{1}{h_{i} r_{1}}+\frac{\ln \left(r_{2} / r_{1}\right)}{k}+\frac{1}{h_{0} r_{2}}} \\
& \frac{Q}{L}=\frac{2 \pi(75-25)}{\frac{1}{30000 \times 0.01375}+\frac{\ln (0.015 / 0.01375)}{43}+\frac{1}{20 \times 0.015}}
\end{aligned}
$$

$$
=94.123 \mathrm{~W} / \mathrm{m}
$$

Hence heat loss per unit length is $94.123 \mathrm{~W} / \mathrm{m}$.

Hence,

Material of manifold - Stainless steel 304

Length $-1200 \mathrm{~mm}$

Diameter (Inner) - 27.5mm

Thermal expansion coefficient $-16 \times 10-6 / \mathrm{K}$

Initial temperature $-750 \mathrm{C}$

Final Temperature $-470 \mathrm{C}$

Maximum Uni-axial thermal stresses - $115.80 \mathrm{MPa}$

Maximum thermal stresses acting on manifold $-79.17 \mathrm{MPa}$

Rate of heat transfer $=398.6 \mathrm{~kJ} / \mathrm{s}$

\begin{tabular}{|c|c|c|}
\hline \multicolumn{3}{|c|}{ Details of "Analysis Settings" } \\
\hline \multirow[t]{5}{*}{$\square$} & \multicolumn{2}{|l|}{ Step Controls } \\
\hline & Number Of Steps & 4. \\
\hline & Current Step Number & 1. \\
\hline & Step End Time & 1. $s$ \\
\hline & Auto Time Stepping & Program Controlled \\
\hline \multirow[t]{2}{*}{$\square$} & \multicolumn{2}{|l|}{ Solver Controls } \\
\hline & Solver Type & Program Controlled \\
\hline \multirow[t]{6}{*}{$\nabla$} & \multicolumn{2}{|l|}{ Radiosity Controls } \\
\hline & Flux Convergence & 1.e-004 \\
\hline & Maximum Iteration & 1000. \\
\hline & Solver Tolerance & 0.1 \\
\hline & Over Relaxation & 0.1 \\
\hline & Hemicube Resolution & 10. \\
\hline \multirow[t]{7}{*}{$\square$} & \multicolumn{2}{|l|}{ Nonlinear Controls } \\
\hline & Heat Convergence & On \\
\hline & --Value & ANSYS Calculated \\
\hline & --Tolerance & $0.5 \%$ \\
\hline & --Minimum Reference & 1.e-006 W \\
\hline & Temperature Convergence & Program Controlled \\
\hline & Line Search & Program Controlled \\
\hline \multirow[t]{4}{*}{$\theta$} & Output Controls & \\
\hline & Calculate Thermal Flux & Yes \\
\hline & Calculate Results At & Equally Spaced Time Points \\
\hline & Number Of Time Points & 1. \\
\hline
\end{tabular}

Heat loss per unit length $=94.123 \mathrm{~W} / \mathrm{m}$.

\section{Steady State Thermal Analysis Of Supply Pipe}

\begin{tabular}{|c|c|c|}
\hline & etails of "Mesh" & \\
\hline$\square$ & Defaults & \\
\hline & Physics Preference & Mechanical \\
\hline & Relevance & 0 \\
\hline$\square$ & Sizing & \\
\hline & Use Advanced Si... & Off \\
\hline & Relevance Center & Coarse \\
\hline & Element Size & Default \\
\hline & Initial Size Seed & Active Assembly \\
\hline & Smoothing & Medium \\
\hline & Transition & Fast \\
\hline & Span Angle Center & Coarse \\
\hline & Minimum Edge L... & $1.57080 \mathrm{~mm}$ \\
\hline$\square$ & Inflation & \\
\hline & Use Automatic Te... & None \\
\hline & Inflation Option & Smooth Transition \\
\hline & Transition Ratio & 0.272 \\
\hline & Maximum Layers & 5 \\
\hline & Growth Rate & 1.2 \\
\hline & Inflation Algorit... & Pre \\
\hline & View Advanced ... & No \\
\hline$\square$ & Advanced & \\
\hline & Shape Checking & Standard Mechanical \\
\hline & Element Midside ... & Program Controlled \\
\hline & Straight Sided El... & No \\
\hline & Number of Retries & Default (4) \\
\hline
\end{tabular}

Following is the analysis on supply pipe and manifold guide.

Fig 10. Analysis Setting
Fig 11. Meshing Details 
10.1 Material Taken Stainless Steel :-

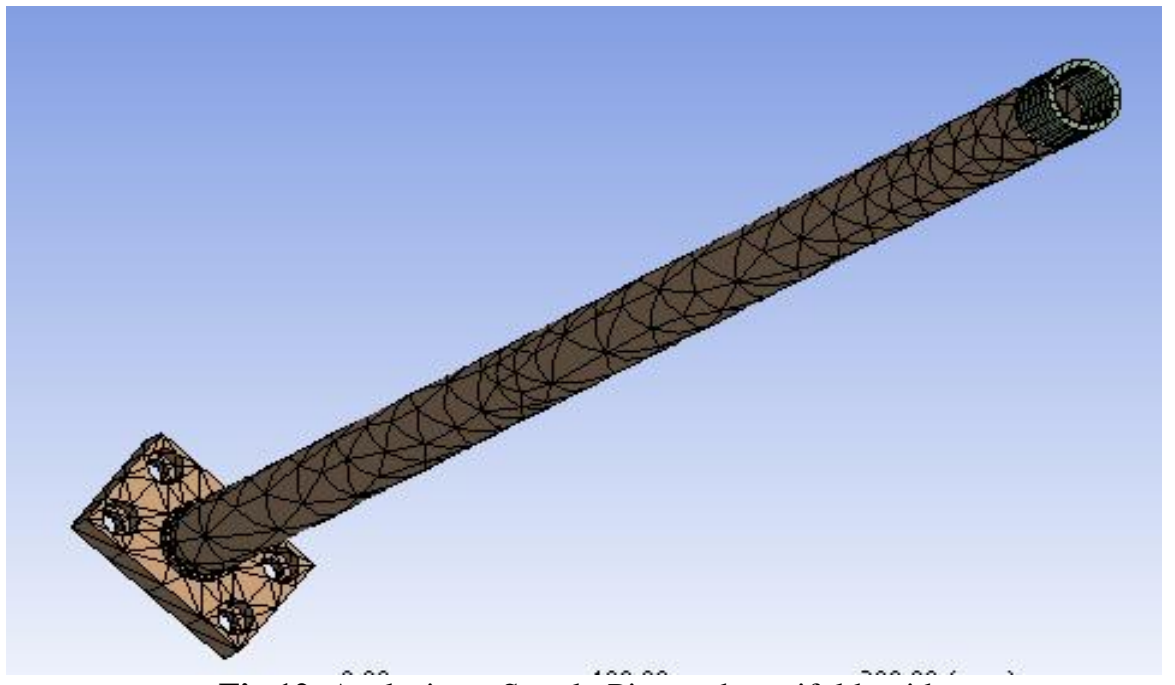

Fig 12. Analysis on Supply Pipe and manifold guide

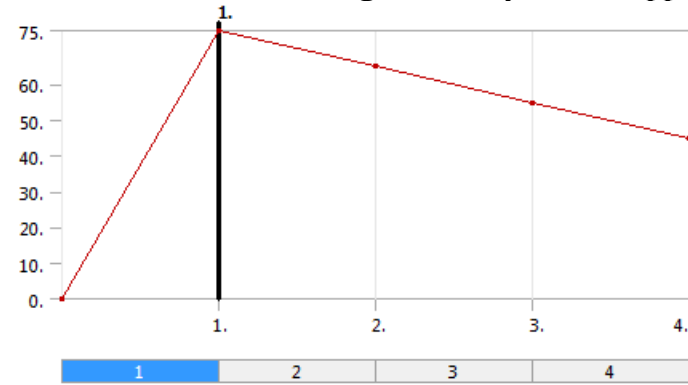

Fig 13 . Input Temprature Graph Against Stages

\begin{tabular}{|l|l|l|l|l} 
& Steps & Time [s] & $\sqrt{\checkmark}$ Temperature $\left[{ }^{\circ} \mathrm{C}\right]$ \\
\hline 1 & 1 & 0. & 0. \\
\hline 2 & 1 & 1. & 75. \\
\hline 3 & 2 & 2. & 65. \\
\hline 4 & 3 & 3. & 55. \\
\hline 5 & 4 & 4. & 45. \\
\hline$\star$ & & & \\
\hline
\end{tabular}

Fig 14 . Temprature Distribution

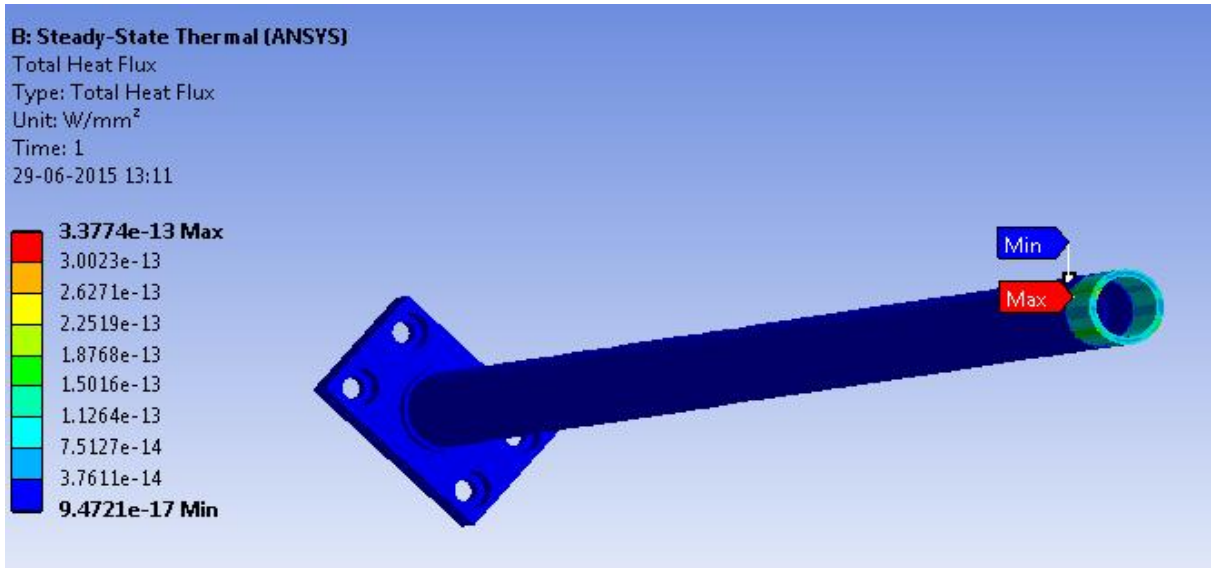

Fig 15 . Stedy State Total Heat Flux Analysis in ANSYS
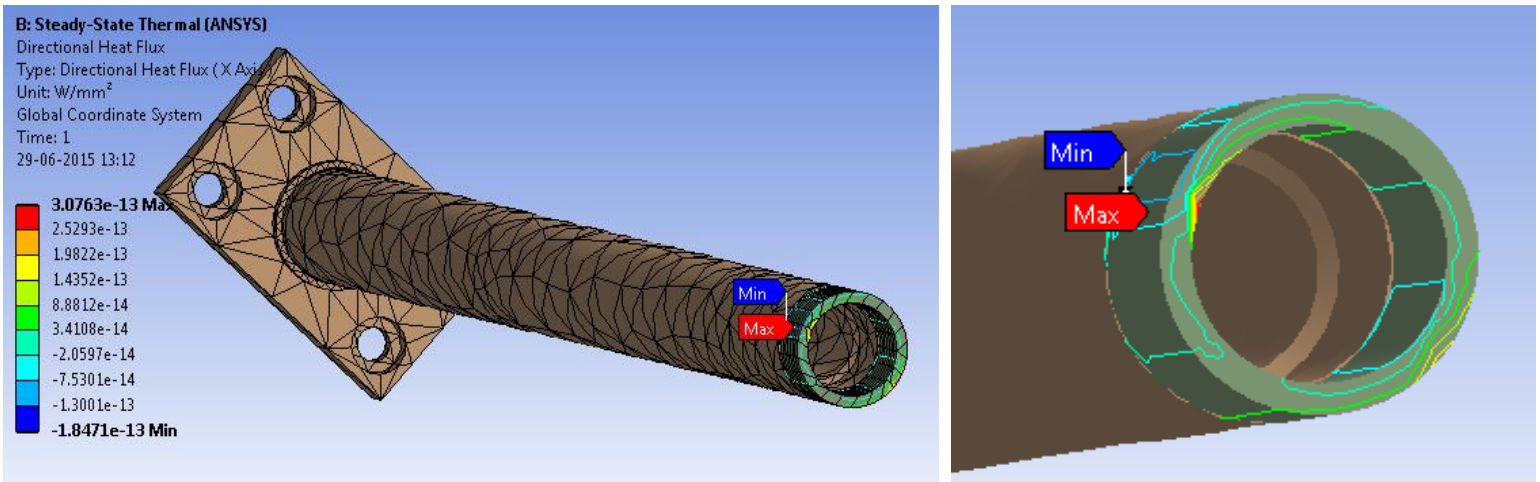

Fig. 16 Steady State Directional Heat Flux Analysis in ANSYS 
10.2 Material Taken is Alluminium Alloy :-

So as to compare results we have taken Alluminium alloy as a material for supply pipes.

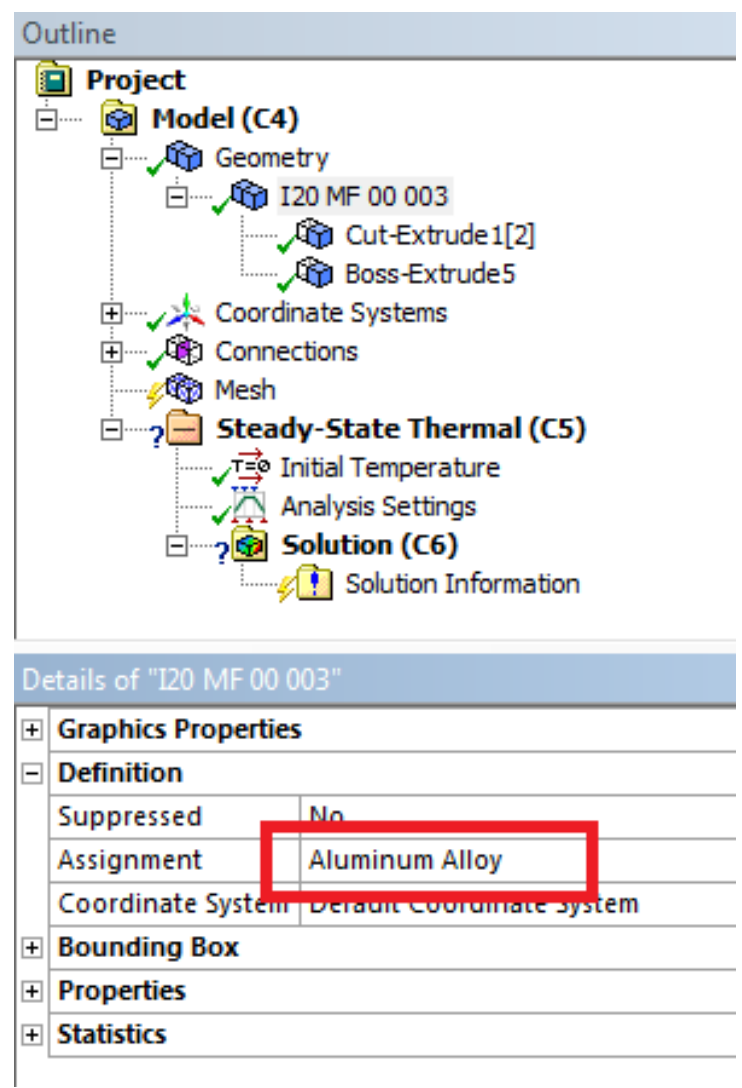

Fig 17. Analysis Inputs

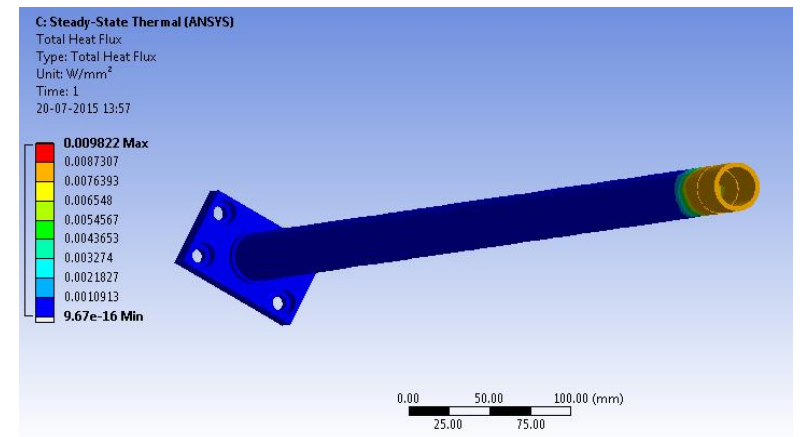

Fig 18 . Stedy State Total Heat Flux Analysis in ANSYS

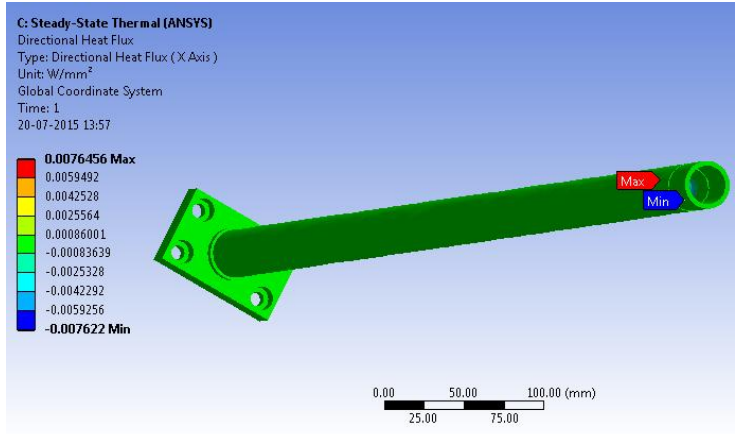

Fig 19. Steady State Directional Heat Flux Analysis YS

\section{Steady State Thermal Analysis Of Distributor In Manifold}
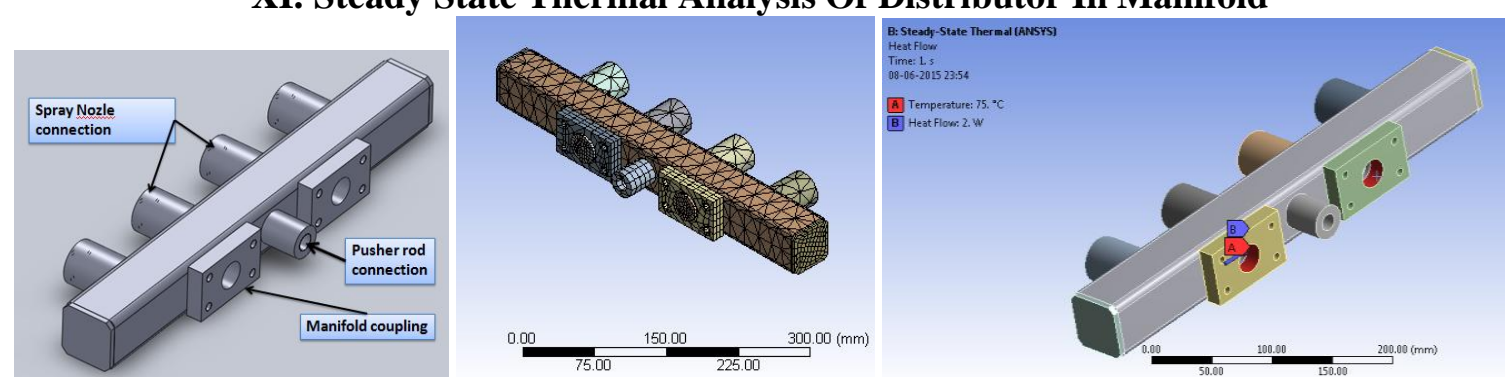

Fig 20 . Extension distributor Fig 21. Steady-State Thermal Analysis Fig 22 . Boundry Condition applied 


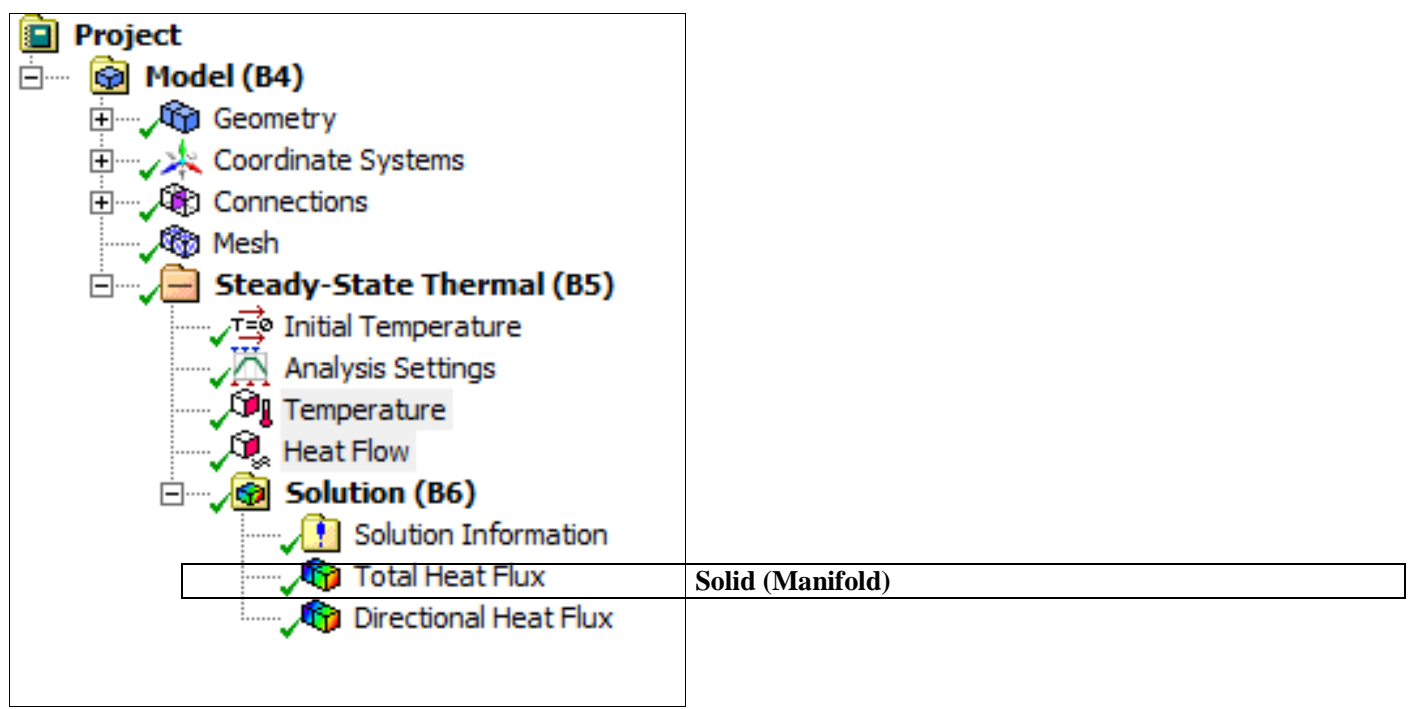

Fig 23 . Parameter Applied

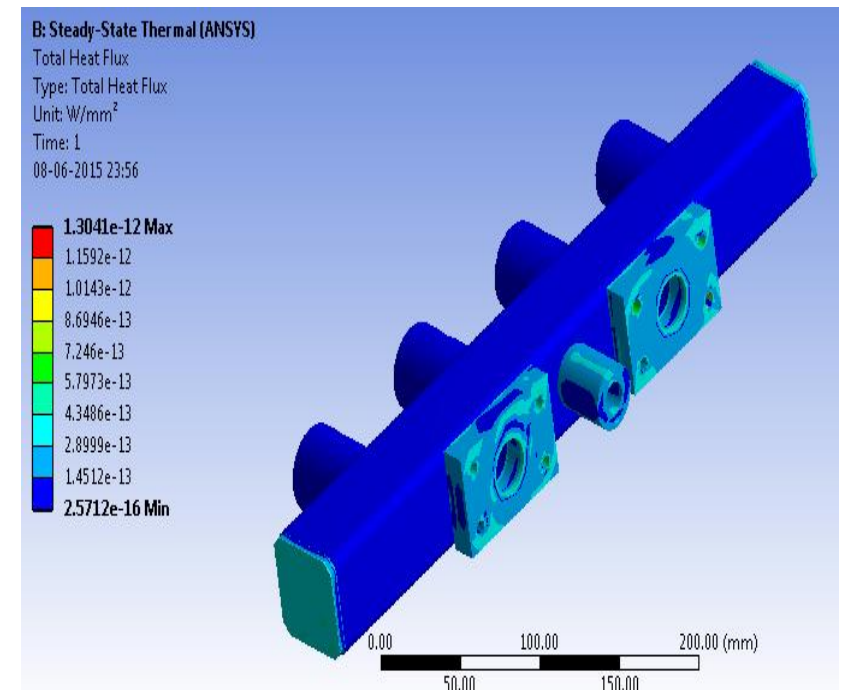

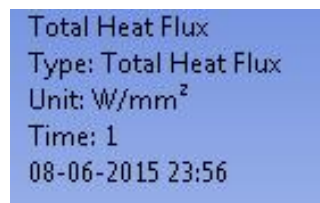

1.3041e-12 Max

$1.1592 \mathrm{e}-12$

$1.0143 \mathrm{e}-12$

$8.6946 \mathrm{e}-13$

$7.246 \mathrm{e}-13$

$5.7973 \mathrm{e}-13$

$4.3486 \mathrm{e}-13$

$2.8999 \mathrm{e}-13$

$1.4512 \mathrm{e}-13$

$2.5712 \mathrm{e}-16 \mathrm{Min}$

Fig 24 . Total Heat Flux in Distributor
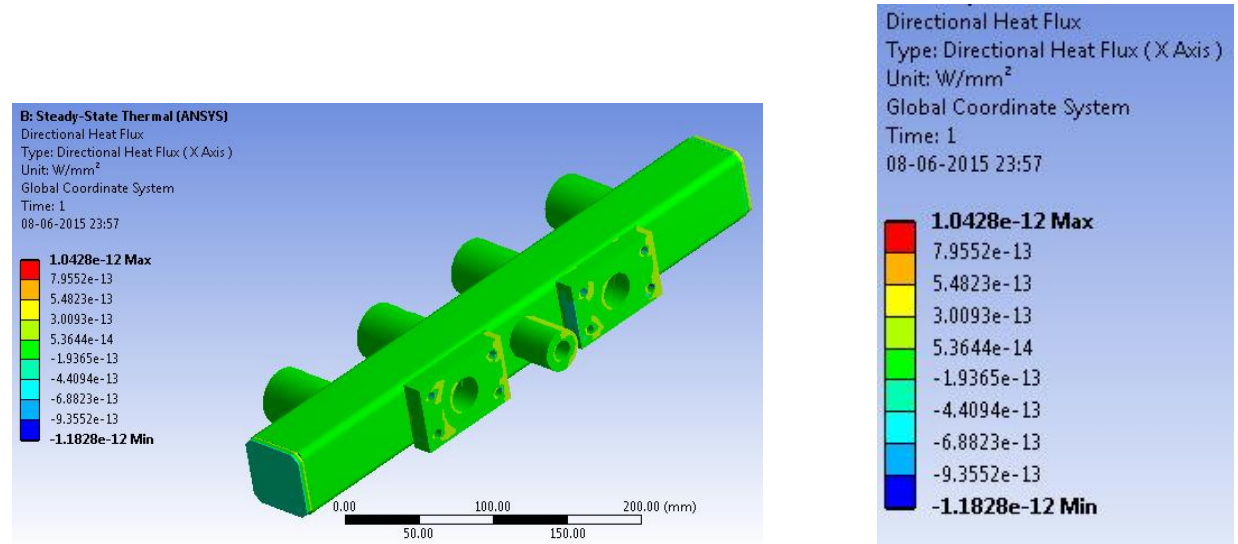

Fig 25 . Directional Heat Flux in Distributor

\section{Results}

The results of this study about thermal stresses in thick-walled pipes (manifold) subjected to fully developed internal flows are given and analysed in this section. The properties of the solids (manifolds) and fluid used in this study are presented in following table 


\begin{tabular}{|c|c|}
\hline \multicolumn{2}{|c|}{ Material : - Steel } \\
\hline Thermal conductivity $(\mathrm{ks})$ & $43 \mathrm{~W} / \mathrm{m} . \mathrm{K}$ \\
\hline Thermal expansion coefficient (a) & $16 \times 10^{-6} / \mathrm{K}$ \\
\hline Modulus of elasticity (E) & $190 \mathrm{GPa}$ \\
\hline Poisson's ratio (v) & 0.3 \\
\hline \multicolumn{2}{|c|}{ Flow } \\
\hline \multicolumn{2}{|c|}{ Flow fluid : - Water } \\
\hline Thermal conductivity $(\mathrm{ks})$ & $0.597 \mathrm{~W} / \mathrm{mK}$ \\
\hline Density (p) & $1000 \mathrm{~kg} / \mathrm{m}^{3}$ \\
\hline Specific heat $(\mathrm{Cp})$ & $4181.8 \mathrm{~J} / \mathrm{KgK}$ \\
\hline Kinematic viscosity $(j j / p)$ & $1.006 \times 10^{-6} \mathrm{~m}^{2} / \mathrm{s}$ \\
\hline \multicolumn{2}{|l|}{ Manifold Specifications } \\
\hline Material of manifold & Stainless steel 304 \\
\hline Length & $1200 \mathrm{~mm}$ \\
\hline Diameter (Inner) & $27.5 \mathrm{~mm}$ \\
\hline Thermal expansion coefficient & $16 \times 10^{-6} / \mathrm{K}$ \\
\hline Initial temperature & $75^{0} \mathrm{C}$ \\
\hline Final Temperature & $47^{0} \mathrm{C}$ \\
\hline Maximum Uni-axial thermal stresses & $115.80 \mathrm{MPa}$ \\
\hline Maximum thermal stresses acting on manifold - & 79.17 MPa \\
\hline Rate of heat transfer & $398.6 \mathrm{~kJ} / \mathrm{s}$ \\
\hline Heat loss & $94.123 \mathrm{~W} / \mathrm{m}$ \\
\hline
\end{tabular}

\section{Conclusion}

From the study of the result mentioned as above. After performing the calculation the output temperature is $45{ }^{\circ} \mathrm{C}$, which is near to the value mentioned output temperature of requirement for washing purpose. This type of Manifold can be installed in industrial washing machine for maintaining temperature difference between input temperature and output temperature

\section{Acknowledgements}

I express my deep sense of gratitude to Dr Rachayya.R.Arakerimath for his valuable guidance.

\section{References}

[1] Pankaj.N.Shrirao,Dr Rajushkumar U Sambhe and Pradeep. R .Bodade. "Convective Heat Transfer Analysis in a Circular Tube with Different Types of Internal Threads of Constant Pitch” IJEAT ISSN: 2249 - 8958, Volume-2, Issue-3, February 2013

[2] Sweta Jain and Alkabani Agrawal, "Coupled Thermal-Structural Finite element analysis for exhaust Manifold of an off road vehicle Diesel engine” IJSCE ISSN: 2231-2307, Volume-3, Issue-4, September 2013

[3] Paresh Patel and Amitesh Paul "Thermal Analysis of Tubular Heat Exchanger By using ANSYS” ISSN: 2278-0181 Vol. 1 Issue 8, October - 2012

[4] Ashish Kumar, Sanjeev Kumar, Utkarsh Nagar, and Avadhesh Yadav."Experimental study of thermal performance of One ended Evacuated tubes for producing Hot Air” Hindawi Publication Corporation,Journal of solar Energy Volume 2013, Article ID 524715, 6 pages

[5] E. Davis, and W. Gill, The Effects of Axial Conduction in The Wall on Heat Transfer with Laminar Flow Int. J. Heat Mass Transfer, Vol. 13, pp. 459-470, 1970

[6] M. Faghri and E.M. Sparrow, "Simultaneous Wall and Fluid Axial Conduction in Laminar Pipe-Flow Heat Transfer", Journal of Heat Transfer, Vol. 102, pp. 58-63, 1980 .

[7] B. Kraishan, "On Conjugated Heat Transfer in a Fully developed Flow", Int. J. Heat Mass Transfer", Vol. 25, No.2, pp. 288-289, 1982.

[8] C. Chang, and W. Chu, "Stresses in a Metal Tube Under Both High Radial Temperature Variation and Internal Pressure", Journal of Applied Mechanics, pp. 101-108, 1954. 